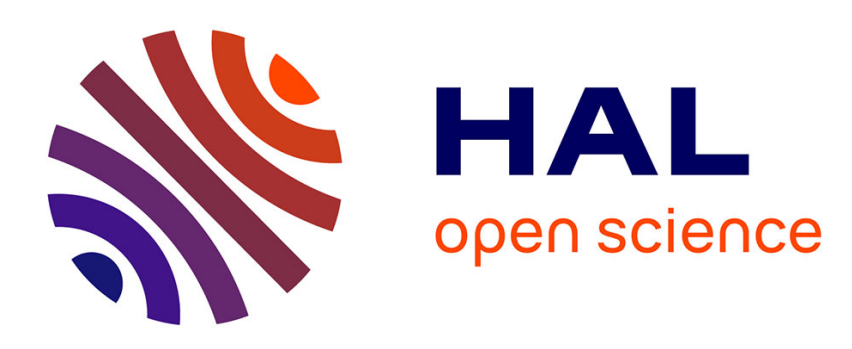

\title{
Modèle analytique compact d'une hétérojonction isotype GaAlAs/GaAs
}

\author{
A. Marty, J.P. Bailbe, G. Rey
}

\section{To cite this version:}

A. Marty, J.P. Bailbe, G. Rey. Modèle analytique compact d'une hétérojonction isotype GaAlAs/GaAs. Revue de Physique Appliquée, 1982, 17 (8), pp.453-464. 10.1051/rphysap:01982001708045300 . jpa-00245022

\section{HAL Id: jpa-00245022 https://hal.science/jpa-00245022}

Submitted on 1 Jan 1982

HAL is a multi-disciplinary open access archive for the deposit and dissemination of scientific research documents, whether they are published or not. The documents may come from teaching and research institutions in France or abroad, or from public or private research centers.
L'archive ouverte pluridisciplinaire HAL, est destinée au dépôt et à la diffusion de documents scientifiques de niveau recherche, publiés ou non, émanant des établissements d'enseignement et de recherche français ou étrangers, des laboratoires publics ou privés. 


\title{
Modèle analytique compact d'une hétérojonction isotype GaAlAs/GaAs
}

\author{
A. Marty, J. P. Bailbe et G. Rey \\ Laboratoire d'Automatique et d'Analyses des Systèmes du C.N.R.S., \\ 7, avenue du Colonel Roche, 31400 Toulouse, France
}

(Reçu le 25 janvier 1982, révisé le 26 avril 1982, accepté le 29 avril 1982)

\begin{abstract}
Résumé. - Dans le cadre de la théorie macroscopique de la diffusion et à partir du diagramme des bandes d'énergie proposé par Anderson, un modèle «à contrôle de la charge " est établi. Celui-ci prend en compte le caractère plus ou moins graduel du changement de largeur de bande interdite à la jonction et permet de préciser la loi de variation du courant avec la tension appliquée par des relations analytiques compactes. Les paramètres de ce modèle sont exprimés en fonction des caractéristiques technologiques et géométriques de la jonction.
\end{abstract}

\begin{abstract}
A charge-control type model, based on the macroscopic theory of the diffusion, and using the energy band diagram proposed by Anderson, is presented. This model accounts for the more or less gradual change of the gap at the junction and allows to present the current variation law with the applied voltage under a compact analytical form. The typical parameters of this model are given as a function of the technological and geometrical characteristics of the junction.
\end{abstract}

1. Introduction. - L'étude de l'hétérojonction isotype, réalisée à partir de matériaux d'affinités électroniques différentes, est d'un grand intérêt pratique. En effet, dans certaines conditions - jonction suffisamment abrupte par exemple - cette structure a un comportement redresseur [1-6].

Plusieurs dispositifs exploitant cette propriété ont été proposés; il s'agit principalement de composants hyperfréquences tels que les diodes de détection $\operatorname{GaAlAs}(\mathrm{N}) / \mathrm{GaAs}(\mathrm{N})$ [4] ou les diodes impatt $\mathrm{Ge}(\mathrm{N}) /$ $\operatorname{GaAs}(\mathrm{N})$ [5], potentiellement très rapides car le transport des charges s'y fait par porteurs majoritaires. Cependant, dans d'autres cas - transistors bipolaires à hétérojonction [7-10], diodes lasers [11, 12] - la nécessité de réaliser des couches de reprise de contact en GaAs sur les zones actives en GaAlAs, fait apparaître des hétérojonctions isotypes parasites qui peuvent engendrer des chutes de tension gênantes susceptibles de limiter les performances des dispositifs.

Dans cet article nous proposons une analyse théorique du comportement électrique de l'hétérojonction isotype $\mathrm{GaAlAs}(\mathrm{N}) / \mathrm{GaAs}(\mathrm{N})$. C'est un problème que nous avons eu à résoudre dans le cadre de travaux plus généraux concernant l'étude et la réalisation de transistors bipolaires à hétérojonction $\mathrm{GaAlAs} / \mathrm{GaAs}$ [7-9].

Le modèle que nous développons est fondé sur la théorie macroscopique de la diffusion et s'appuie sur le diagramme des bandes d'énergie proposé par Anderson [1]. Nous ne considérerons que le cas des diodes " courtes" idéales dans lesquelles les recombinaisons en volume et les recombinaisons à l'interface peuvent être négligées. Par contre, le caractère plus ou moins graduel de la variation de largeur de bande interdite sera pris en compte.

2. Analyse théorique. - 2.1 ReLATIONS GÉNÉRALES. - Pour décrire entièrement le comportement d'une structure réalisée dans un cristal semiconducteur présentant des caractéristiques non uniformes (densités effectives d'état, affinité électronique, largeur de bande interdite, dopage... fonctions des coordonnées de position), les équations de transport de charge, qui constituent le point de départ de bien des calculs, s'écrivent dans le cadre d'un modèle de diffusion $[13,14]$ :

$$
\begin{aligned}
J_{\mathrm{n}}=q D_{\mathrm{n}} n & {\left[-\frac{1}{U_{\mathrm{T}}} \frac{\mathrm{d}}{\mathrm{d} w}(V+\chi)+\frac{\mathrm{d}}{\mathrm{d} w}\left(\ln \frac{n}{N_{\mathrm{C}}}\right)\right] } \\
J_{\mathrm{p}}=q D_{\mathrm{p}} p & {\left[-\frac{1}{U_{\mathrm{T}}} \frac{\mathrm{d}}{\mathrm{d} w}(V+\chi)-\right.} \\
& \left.-\frac{\mathrm{d}}{\mathrm{d} w}\left(\ln \frac{p}{N_{\mathrm{v}}}\right)-\frac{1}{k T} \frac{\mathrm{d} E_{\mathrm{g}}}{\mathrm{d} w}\right]
\end{aligned}
$$

où $V$ désigne le potentiel électrostatique, $J_{\mathbf{n}}$ et $J_{\mathrm{p}}$ les densités de courants d'électrons et de trous, $\chi$ 
l'affinité électronique, $E_{\mathrm{g}}$ la largeur de bande interdite, $N_{\mathrm{C}}$ et $N_{\mathrm{v}}$ les densités effectives d'état dans les bandes de conduction et de valence, $n$ et $p$ les concentrations d'électrons et de trous, $D_{\mathrm{n}}$ et $D_{\mathrm{p}}$ les constantes de diffusion, $U_{\mathrm{T}}$ l'unité de tension thermodynamique et $w$ l'abscisse de position.

A partir de (1) et (2) on peut établir très simplement toute une série de résultats que nous allons résumer ci-après sans démonstration, en renvoyant le lecteur aux références bibliographiques [7] et [15].

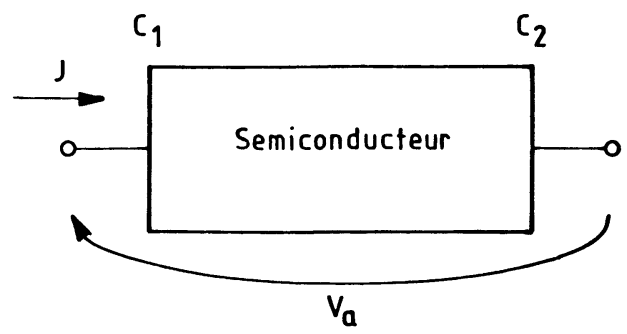

Fig. 1. - Notations.

[Notations.]

Si l'on considère un barreau muni de deux contacts ohmiques $C_{1}$ et $C_{2}$ (Fig. 1), la différence de potentiel que supporte le semiconducteur à l'équilibre thermodynamique (tension de diffusion) est donnée par

$$
V_{\mathrm{D}}=\frac{\chi_{\mathrm{C}_{1}}-\chi_{\mathrm{C}_{2}}}{q}+U_{\mathrm{T}} \ln \left(\frac{\bar{n}}{N_{\mathrm{C}}}\right)_{\mathrm{C}_{2}}-U_{\mathrm{T}} \ln \left(\frac{\bar{n}}{N_{\mathrm{C}}}\right)_{\mathrm{C}_{1}}
$$

ou encore

$$
\begin{aligned}
V_{\mathrm{D}}=\frac{\chi_{\mathrm{C}_{1}}-\chi_{\mathrm{C}_{2}}}{q} & +\frac{E_{\mathrm{gC}_{1}}-E_{\mathrm{gC}_{2}}}{q}+ \\
& +U_{\mathrm{T}} \ln \left(\frac{\bar{p}}{N_{\mathrm{V}}}\right)_{\mathrm{C}_{1}}-U_{\mathrm{T}} \ln \left(\frac{\bar{p}}{N_{\mathrm{V}}}\right)_{\mathrm{C}_{2}}
\end{aligned}
$$

$\bar{n}$ et $\bar{p}$ représentent les concentrations de porteurs à l'équilibre thermodynamique.

Par ailleurs, lorsqu'on applique entre ces contacts ohmiques une tension extérieure $V_{\mathrm{a}}$, on démontre que :

$$
\frac{V_{\mathrm{a}}}{U_{\mathrm{T}}}=\int_{\mathrm{C}_{1}}^{\mathrm{C}_{2}} \frac{J_{\mathrm{n}}}{q D_{\mathrm{n}} n} \mathrm{~d} w=\int_{\mathrm{C}_{1}}^{\mathrm{C}_{2}} \frac{J_{\mathrm{p}}}{q D_{\mathrm{p}} p} \mathrm{~d} w .
$$

Enfin la relation générale

$$
\frac{p}{n_{\mathrm{i}}^{2}} \frac{J_{\mathrm{n}}}{q D_{\mathrm{n}}}-\frac{n}{n_{\mathrm{i}}^{2}} \frac{J_{\mathrm{p}}}{q D_{\mathrm{p}}}=\frac{\mathrm{d}}{\mathrm{d} w}\left(\frac{p n}{n_{\mathrm{i}}^{2}}\right)
$$

permet de relier la valeur du produit pn des concentrations de porteurs en un point d'abscisse $w$ à la tension appliquée; il vient :

$\ln \left(\frac{p n}{n_{\mathrm{i}}^{2}}\right)_{w}=\frac{V_{\mathrm{a}}}{U_{\mathrm{T}}}-\int_{w}^{\mathrm{C}_{2}} \frac{J_{\mathrm{n}}}{q D_{\mathrm{n}} n} \mathrm{~d} w-\int_{\mathrm{C}_{1}}^{w} \frac{J_{\mathrm{p}}}{q D_{\mathrm{p}} p} \mathrm{~d} w$

où $n_{\mathrm{i}}$ désigne la densité intrinsèque au point considéré.
Ces résultats sont valables quelles que soient les particularités de la structure limitée par les contacts $C_{1}$ et $C_{2}$ et aucune hypothèse restrictive n'a été émise autre que celles qu'implique l'usage de (1) et (2). Lorsque les densités de porteurs $n$ et $p$ obéissent à la statistique ue Maxwell-Boltzmann: les deux termes qui apparaissent sous forme intégrale dans (7) représentent les variations des pseudo-niveaux de Fermi des électrons et des trous entre les bornes d'intégration; en effet, on peut écrire :

$$
\begin{aligned}
& \int_{x_{1}}^{x_{2}} \frac{J_{\mathrm{n}}}{q D_{\mathrm{n}} n} \mathrm{~d} w=\frac{E_{\mathrm{Fn}}\left(x_{2}\right)-E_{\mathrm{Fn}}\left(x_{1}\right)}{k T} \\
& \int_{x_{1}}^{x_{2}} \frac{J_{\mathrm{p}}}{q D_{\mathrm{p}} p} \mathrm{~d} w=\frac{E_{\mathrm{Fp}}\left(x_{2}\right)-E_{\mathrm{Fp}}\left(x_{1}\right)}{k T} .
\end{aligned}
$$

Si on néglige l'influence des recombinaisons, on montre que l'efficacité d'injection, définie par le rapport $J_{n} / J_{p}$ des densités de courants d'électrons et de trous, est donnée par :

$$
\gamma=J_{\mathrm{n}} / J_{\mathrm{p}}=\int_{\mathrm{C}_{1}}^{\mathrm{C}_{2}} \frac{n}{n_{\mathrm{i}}^{2} D_{\mathrm{p}}} \mathrm{d} w / \int_{\mathrm{C}_{1}}^{\mathrm{C}_{2}} \frac{p}{n_{\mathrm{i}}^{2} D_{\mathrm{n}}} \mathrm{d} w .
$$

Bien entendu, à l'ensemble de relations générales qui vient d'être rappelé, il convient d'adjoindre, pour décrire entièrement le comportement électrique de la structure, d'une part les équations de continuité

$$
\frac{\mathrm{d} J_{\mathrm{n}}}{\mathrm{d} w}=\frac{\mathrm{d} J_{\mathrm{p}}}{\mathrm{d} w}=0
$$

et d'autre part, l'équation de l'électrostatique

$$
\frac{\mathrm{d}^{2} V}{\mathrm{~d} w^{2}}=-\frac{q}{\varepsilon}\left(p-n+N_{\mathrm{D}}\right)-\frac{1}{\varepsilon} \frac{\mathrm{d} V}{\mathrm{~d} w} \frac{\mathrm{d} \varepsilon}{\mathrm{d} w}
$$

où $\varepsilon$ représente la permittivité diélectrique du matériau semiconducteur.

2.2 CAS DE L'HÉTÉROJONCTION ISOTYPE $\mathrm{GaAlAs}(\mathrm{N}) / \mathrm{GaAs}(\mathrm{N})$. - Les diagrammes de la figure 2 illustrent la situation des bandes d'énergie à l'équilibre thermodynamique des hétérojonctions GaAlAs(N)/GaAs(N) abrupte et graduelle. Pour distinguer les deux régions $\mathrm{N}$, nous avons affecté l'indice « 1 » aux paramètres relatifs à la zone qui présente la plus faible largeur de bande interdite et l'indice « 2 " aux paramètres de la zone opposée; $W_{1}$ et $W_{2}$ désignent les extensions de la zone de transition de part et d'autre de la jonction métallurgique $J$.

2.2.1 Tension de diffusion. - La courbure des bandes entre $W_{1}$ et $W_{2}$ fait apparaître une zone d'accumulation de très faible épaisseur entre $W_{1}$ et $J$ - c'est-à-dire du côté où $E_{\mathrm{g}}$ est le plus petit - et une zone dépeuplée beaucoup plus étendue entre $J$ et $W_{2}$. Cette dissymétrie de la charge d'espace se retrouve au 

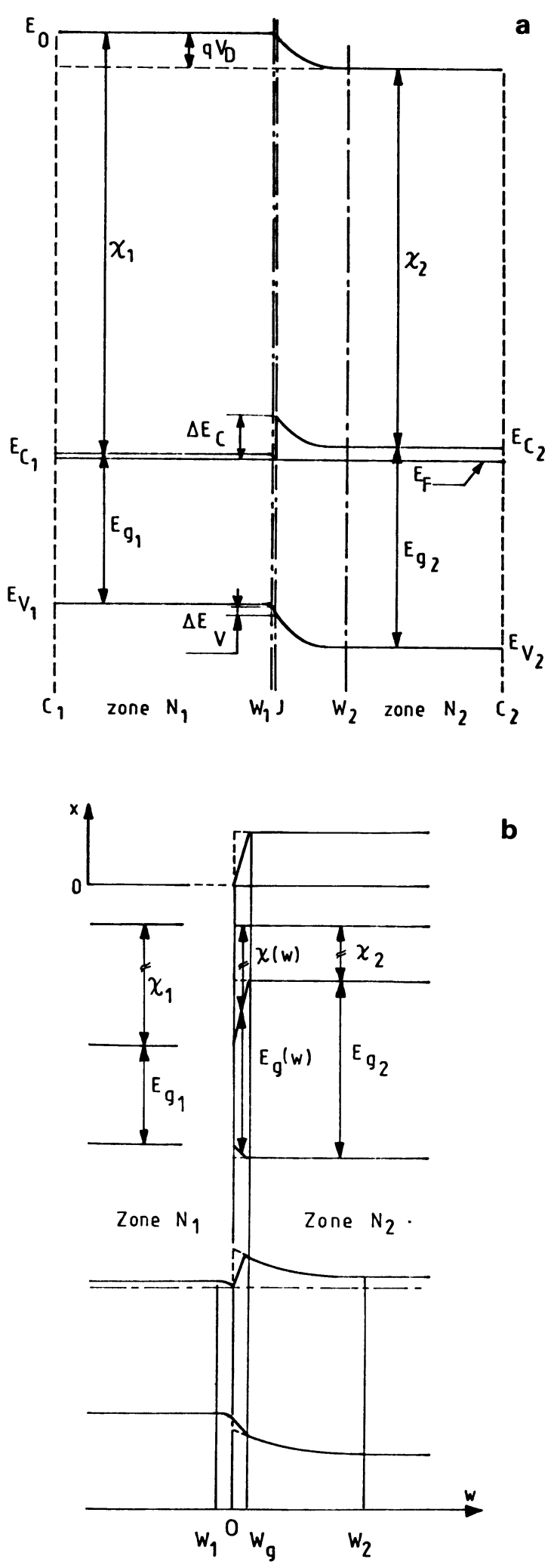

Fig. 2. - Diagrammes des bandes d'énergie des hétérojonctions $\mathrm{GaAs}\left(\mathrm{N}_{1}-10^{17} \mathrm{~cm}^{-3}\right) / \mathrm{Ga}_{0,6} \mathrm{Al}_{0,4} \mathrm{As}\left(\mathrm{N}_{2}-\right.$ $10^{16} \mathrm{~cm}^{-3}$ ) à l'équilibre thermodynamique : a) jonction abrupte; $b$ ) jonction graduelle.

[Energy band diagrams of a $\operatorname{GaAs}\left(\mathrm{N}_{1}-10^{17} \mathrm{~cm}^{-3}\right) /$ $\mathrm{Ga}_{0.6} \mathrm{Al}_{0.4} \mathrm{As}\left(\mathrm{N}_{2}-10^{16} \mathrm{~cm}^{-3}\right)$ heterojunctions at equilibrium : a) abrupt heterojunction; b) graded-gap heterojunction.] niveau de la répartition de la différence de potentiel que supporte la zone de transition : l'essentiel de celle-ci est localisé entre $\mathbf{J}$ et $\mathbf{W}_{2}$, dans la zone à fort « gap ». La tension de diffusion $V_{\mathrm{D}}$, définie par la relation (3), s'écrit ici :

$$
V_{\mathrm{D}}=\frac{\chi_{1}-\chi_{2}}{q}+U_{\mathrm{T}} \ln \left(\frac{\mathrm{N}_{\mathrm{D}_{2}}}{\mathrm{~N}_{\mathrm{D}_{1}}} \cdot \frac{\mathrm{N}_{\mathrm{C}_{1}}}{\mathrm{~N}_{\mathrm{C}_{2}}}\right)
$$

Cette expression montre que $V_{\mathrm{D}}$ a deux origines : l'une liée à la différence des dopages $-N_{\mathrm{D}_{1}}, N_{\mathrm{D}_{2}}-$ (peu importante dans le cas d'une jonction isotype), l'autre liée à la dissymétrie des matériaux (écart entre les affinités électroniques $-\chi_{1}, \chi_{2}$ - essentiellement). Selon que la tension extérieure appliquée ajoutera ou retranchera ses effets à ceux de la tension de diffusion, l'hétérojonction sera en polarisation inverse ou directe, mais dans tous les cas l'essentiel du courant sera véhiculé par les électrons qui sont largement majoritaires sur la plus grande partie de la structure.

Notons qu'une hétérojonction du type $\operatorname{GaAlAs}(\mathrm{P}) /$ $\mathrm{GaAs}(\mathrm{P})$ dont le diagramme des bandes est reproduit sur la figure 3 n'aurait pas un comportement redresseur sensible. En effet, l'« affinité des trous" $\left(E_{\mathrm{g}}+\chi\right)$ est à peu de choses près la même dans le GaAlAs et dans le GaAs et, par conséquent, la tension de diffusion est extrêmement faible puisque essentiellement liée

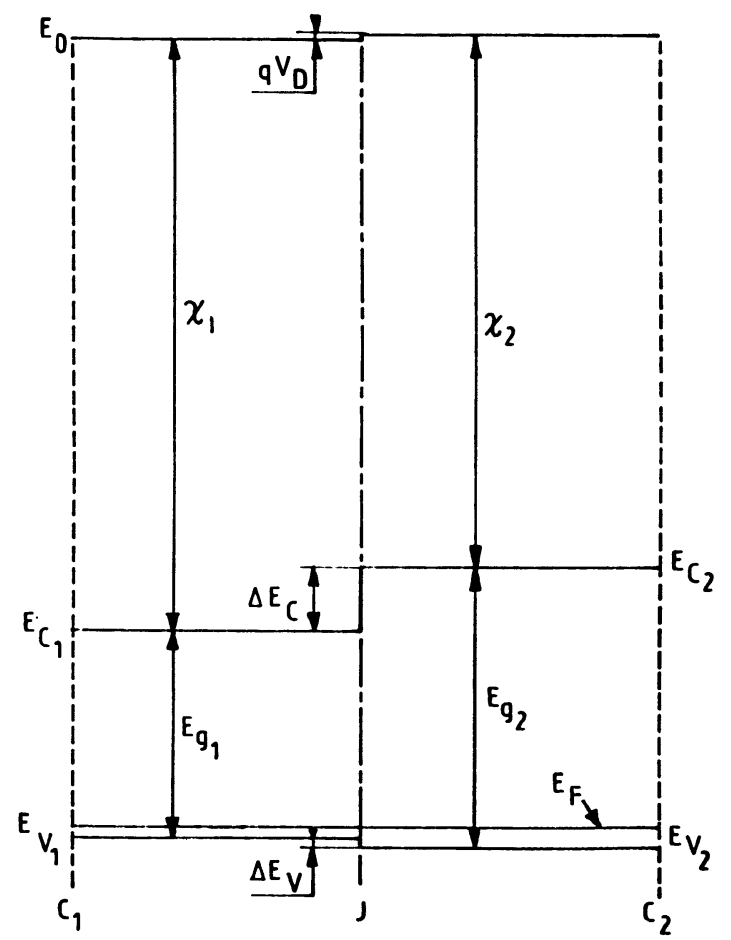

Fig. 3. - Diagramme des bandes d'énergie de l'hétérojonction $\operatorname{GaAs}\left(\mathrm{P}_{1}-10^{18} \mathrm{~cm}^{-3}\right) / \mathrm{Ga}_{0,6} \mathrm{Al}_{0,4} \mathrm{As}\left(\mathrm{P}_{2}-10^{17} \mathrm{~cm}^{-3}\right)$ à l'équilibre thermodynamique.

[Energy band diagram of a $\operatorname{GaAs}\left(\mathrm{P}_{1}-10^{18} \mathrm{~cm}^{-3}\right) /$ $\mathrm{Ga}_{0.6} \mathrm{Al}_{0.4} \mathrm{As}\left(\mathrm{P}_{2}-10^{17} \mathrm{~cm}^{-3}\right)$ heterojunction at equilibrium.] 
à la différence des dopages. Il n'y a donc pratiquement pas de zone de transition, et le comportement de la structure est de type ohmique quelle que soit la polarité de la tension appliquée.

Le diagramme des bandes d'une hétérojonction isotype N-N (Fig. 2) fait apparaître dans la zone à grand " gap » une pointe importante sur la bande de conduction tout à fait analogue à celle qui apparaît dans une structure anisotype [7]. La présence de ce "spike " joue, dans le comportement de l'hétérojonction, un rôle déterminant que nous préciserons.

2.2.2 Calcul $d u$ produit pn. - Il est essentiel de remarquer que, dans la zone de "spike ", la concentration d'électrons est considérablement réduite par rapport à ce qu'elle est dans les deux régions quasineutres et $a$ fortiori dans la zone d'accumulation. Dans ces conditions, les relations

$$
\int_{\mathrm{J}}^{\mathrm{W}_{2}} \frac{J_{\mathrm{n}}}{q D_{\mathrm{n}} n} \mathrm{~d} w \simeq \int_{\mathrm{C}_{1}}^{\mathrm{C}_{2}} \frac{J_{\mathrm{n}}}{q D_{\mathrm{n}} n} \mathrm{~d} w=\frac{V_{\mathrm{a}}}{U_{\mathrm{T}}}
$$

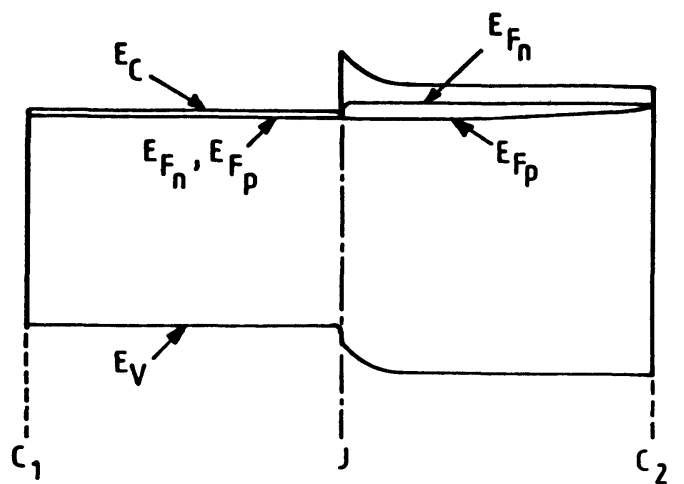

a) $v_{a}=0,1 v_{j} J_{n}=19,4 \mathrm{~A} / \mathrm{cm}^{2}$

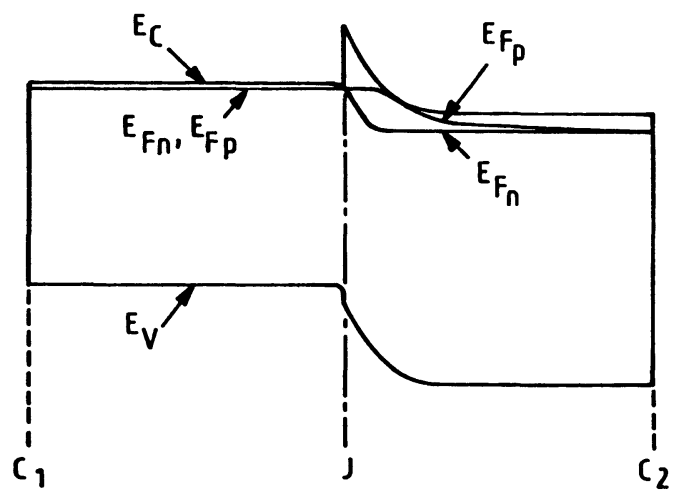

b) $v_{a}=-0,3 V ; J_{n}=1,3 \mathrm{~A} / \mathrm{cm}^{2}$

Fig. 4. - Position des pseudo-niveaux de Fermi des porteurs dans l'hétérojonction abrupte $\operatorname{GaAs}\left(\mathrm{N}_{1}-10^{17} \mathrm{~cm}^{-3}\right)$ / $\mathrm{Ga}_{0,6} \mathrm{Al}_{0.4}^{\prime} \mathrm{As}\left(\mathrm{N}_{2}-10^{16} \mathrm{~cm}^{-3}\right): a$ ) en régime de fonctionnement direct ; $b$ ) en régime de fonctionnement inverse.

[Position of quasi-Fermi levels for carriers in abrupt $\mathrm{GaAs}\left(\mathrm{N}_{1}-10^{17} \mathrm{~cm}^{-3}\right) / \mathrm{Ga}_{0.6} \mathrm{Al}_{0.4} \mathrm{As}\left(\mathrm{N}_{2}-10^{16} \mathrm{~cm}^{-3}\right)$ heterojunction $: a$ ) forward bias; $b$ ) reverse bias.] ou

$\int_{\mathrm{C}_{1}}^{\mathrm{J}} \frac{J_{\mathrm{n}}}{q D_{\mathrm{n}} n} \mathrm{~d} w+\int_{\mathrm{W}_{2}}^{\mathrm{C}_{2}} \frac{J_{\mathrm{n}}}{q D_{\mathrm{n}} n} \mathrm{~d} w \ll \int_{\mathrm{J}}^{\mathrm{W}_{2}} \frac{J_{\mathrm{n}}}{q D_{\mathrm{n}} n} \mathrm{~d} w$

sont satisfaites, ce qui implique que le pseudo-niveau de Fermi des électrons est pratiquement "plat » dans toute la structure sauf à la traversée de la zone de « spike » (Fig. 4).

Pour sa part, la population de trous se raréfie d'autant plus que la largeur de bande interdite est grande ; il est alors permis d'écrire :

$$
\int_{\mathrm{C}_{1}}^{\mathrm{J}} \frac{J_{\mathrm{p}}}{q D_{\mathrm{p}} p} \mathrm{~d} w \ll \int_{\mathrm{J}}^{\mathrm{C}_{2}} \frac{J_{\mathrm{p}}}{q D_{\mathrm{p}} p} \mathrm{~d} w \simeq \frac{V_{\mathrm{a}}}{U_{\mathrm{T}}} .
$$

Le pseudo-niveau de Fermi des trous est donc constant entre $C_{1}$ et $J$ (Fig. 4).

La figure 5, qui reproduit les résultats obtenus par la résolution numérique complète des équations (1), (2), (11) et $(12)[14,16$, permet de juger du degré de validité des fortes inégalités (14) à (16).

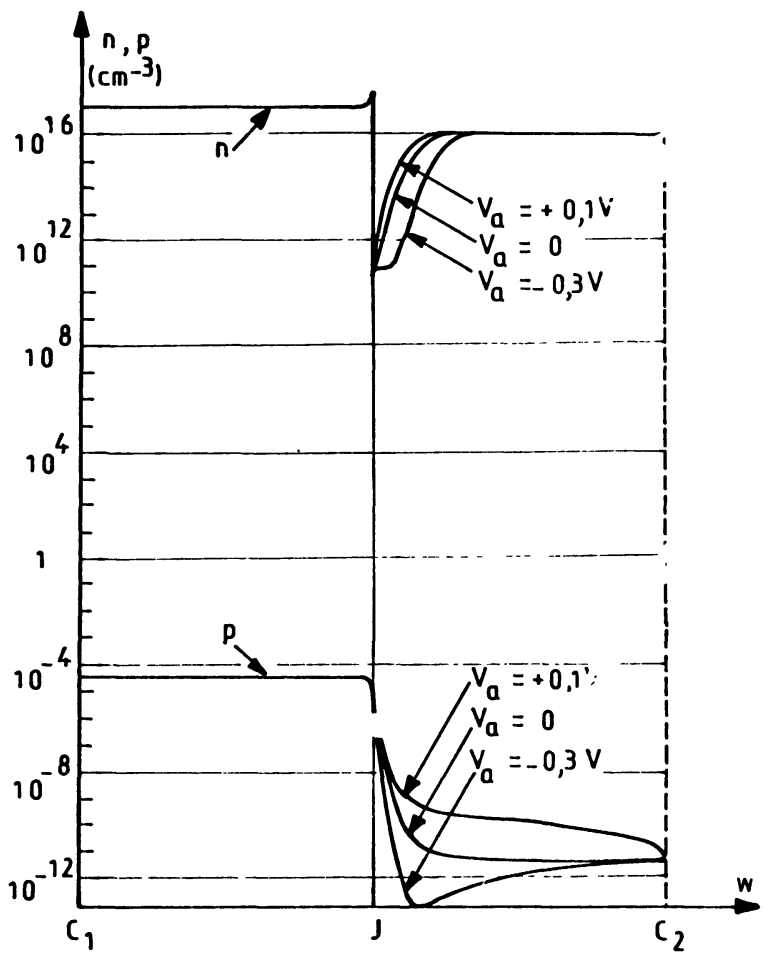

Fig. 5. - Répartition des concentrations de porteurs $n(w)$ et $p(w)$ dans l'hétérojonction $\operatorname{GaAs}\left(\mathrm{N}_{1}-10^{17} \mathrm{~cm}^{-3}\right) /$ $\mathrm{Ga}_{0,6} \mathrm{Al}_{0,4} \mathrm{As}\left(\mathrm{N}_{2}-10^{16} \mathrm{~cm}^{-3}\right)$.

[Distributions of carriers concentration $n(w)$ and $p(w)$ in a $\operatorname{GaAs}\left(\mathrm{N}_{1}-10^{17} \mathrm{~cm}^{-3}\right) / \mathrm{Ga}_{0.6} \mathrm{Al}_{0.4} \mathrm{As}\left(\mathrm{N}_{2}-10^{16} \mathrm{~cm}^{-3}\right)$ heterojunction.]

Dès lors, d'après la relation (7), le produit pn des concentrations d'électrons et de trous reste pratiquement constant et égal à $n_{\mathrm{i}}^{2}$ entre $\mathrm{C}_{1}$ et $\mathrm{J}$, c'est-à-dire 
dans toute la région $\mathrm{N}_{1}$; en particulier, à la jonction on peut écrire :

$$
\left(\frac{p n}{n_{\mathrm{i}}^{2}}\right)_{\mathrm{J}} \simeq 1
$$

tout comme ce serait le cas pour un contact ohmique. Par ailleurs, toujours d'après (7) et compte tenu de (14), (15) et (16), l'évaluation du produit $p n$ à la limite $W_{2}$ de la zone de déplétion conduit à :

$$
\left(\frac{p n}{n_{\mathrm{i}}^{2}}\right)_{\mathrm{w}_{2}} \simeq \exp \left(\frac{V_{\mathrm{a}}}{U_{\mathrm{T}}}\right) \exp \left[-\int_{\mathrm{J}}^{\mathrm{W}_{2}} \frac{J_{\mathrm{p}}}{q D_{\mathrm{p}} p} \mathrm{~d} w\right] .
$$

On est ainsi, pour ce qui concerne la région $\mathrm{N}_{2}$, dans une situation tout à fait analogue à celle rencontrée dans le cas d'une diode Schottky [15].

Pour des tensions de polarisation directes, la concentration des trous dans la zone de transition comprise entre $\mathbf{J}$ et $\mathbf{W}_{\mathbf{2}}$ prend des valeurs supérieures à celles observées dans la région quasi-neutre correspondante (entre $\mathrm{W}_{2}$ et $\mathrm{C}_{2}$ ). Dans ces conditions,

$$
\int_{\mathrm{J}}^{\mathrm{W}_{2}} \frac{J_{\mathrm{p}}}{q D_{\mathrm{p}} p} \mathrm{~d} w \ll \frac{V_{\mathrm{a}}}{U_{\mathrm{T}}}
$$

et

$$
\left(\frac{p n}{n_{\mathrm{i}}^{2}}\right)_{\mathrm{w}_{2}} \simeq \exp \left(\frac{V_{\mathrm{a}}}{U_{\mathrm{T}}}\right)
$$

En polarisation inverse, la forte inégalité (19) n'est plus satisfaisante mais, compte tenu des valeurs extrêmement faibles de $\exp \left(V_{\mathrm{a}} / U_{\mathrm{T}}\right)$, on peut écrire :

$$
\left(\frac{p n}{n_{\mathrm{i}}^{2}}\right)_{\mathrm{W}_{2}} \ll 1
$$

2.2.3 Efficacité d'injection. - Les fortes inégalités entre les concentrations de porteurs dans les différentes zones de l'hétérojonction permettent de simplifier l'expression générale de l'efficacité d'injection (10). Ainsi tenant compte de ce que

$$
E_{\mathrm{g}}(\mathrm{GaAlAs})>E_{\mathrm{g}}(\mathrm{GaAs})
$$

implique $\left(n_{\mathrm{i}}^{2}\right)_{2} \ll\left(n_{\mathrm{i}}^{2}\right)_{1}$, on aboutit à :

$$
\gamma=J_{\mathrm{n}} / J_{\mathrm{p}} \simeq \int_{\mathrm{W}_{2}}^{\mathrm{C}_{2}} \frac{n}{D_{\mathrm{p}}} \mathrm{d} w / \int_{\mathrm{J}}^{\mathrm{C}_{2}} \frac{p}{D_{\mathrm{n}}} \mathrm{d} w .
$$

La très faible valeur de la population des trous comparée à celle de la population d'électrons conduit à des valeurs considérables de $\gamma$ : dès lors le courant intrinsèque $J_{\mathrm{p}}$ est de faible amplitude et on peut le négliger par rapport à celui lié aux recombinaisons toujours présentes dans un dispositif réel.

L'ensemble de ces relations générales va nous permettre d'établir simplement l'équation de la caractéristique statique $(J, V)$.
2.3 CARACTÉRISTIQUE COURANT-TENSION DE L'HÉTÉROJONCTION GaAlAs(N)/GaAs(N). - L'intégration de la relation générale (6) étendue à la région quasineutre $\mathrm{W}_{2}-\mathrm{C}_{2}$ conduit à :

$\int_{\mathrm{W}_{2}}^{\mathrm{C}_{2}} \frac{J_{\mathrm{p}} n}{q n_{\mathrm{i}}^{2} D_{\mathrm{p}}} \mathrm{d} w-\int_{\mathrm{W}_{2}}^{\mathrm{C}_{2}} \frac{J_{\mathrm{n}} p}{q n_{\mathrm{i}}^{2} D_{\mathrm{n}}} \mathrm{d} w=\left(\frac{p n}{n_{\mathrm{i}}^{2}}\right)_{\mathrm{w}_{2}}-1$

soit, compte tenu de (20) et (21) :

$\int_{\mathrm{W}_{2}}^{\mathrm{C}_{2}} \frac{J_{\mathrm{p}} n}{q n_{\mathrm{i}}^{2} D_{\mathrm{p}}} \mathrm{d} w-\int_{\mathrm{W}_{2}}^{\mathrm{C}_{2}} \frac{J_{\mathrm{n}} p}{q n_{\mathrm{i}}^{2} D_{\mathrm{n}}} \mathrm{d} w \simeq \exp \left(\frac{V_{\mathrm{a}}}{U_{\mathrm{T}}}\right)-1$.

Si on ignore les recombinaisons, l'élimination de $J_{\mathrm{p}}$ par usage de la relation (22) conduit à :

$$
J_{\mathrm{n}} \simeq \frac{\exp \left(\frac{V_{\mathrm{a}}}{U_{\mathrm{T}}}\right)-1}{\int_{\mathrm{J}}^{\mathrm{W}_{2}} \frac{p}{q n_{\mathrm{i}}^{2} D_{\mathrm{n}}} \mathrm{d} w} .
$$

On peut observer que cette relation est tout à fait analogue à celle qui serait obtenue dans le cas d'une homojonction dont la région $\mathbf{P}$ se situerait entre $\mathbf{J}$ et $W_{2}$ et que, si l'intégrale de la concentration des trous qui figure au dénominateur de l'expression (25) était indépendante du niveau de courant, la caractéristique de l'hétérojonction isotype serait semblable à celle d'une jonction $\mathrm{P}-\mathrm{N}$; la densité du courant de saturation serait toutefois beaucoup plus grande en raison de la faible valeur de l'intégrale qui porte ici sur les concentrations des trous minoritaires et non sur les porteurs majoritaires.

Notons que ce résultat souligne le rôle déterminant joué par le "spike " puisque l'intégrale qui figure au dénominateur de (25) concerne les concentrations de trous dont les valeurs sont directement liées à l'amplitude du "spike».

Pour préciser un peu mieux la loi de variation de $J_{\mathrm{n}}$ en fonction de la tension appliquée $V_{\mathrm{a}}$, il convient de déterminer la loi de répartition des trous dans la zone dépeuplée. Tenant compte de ce que d'une part l'essentiel de la variation de potentiel électrostatique que supporte la jonction se situe dans la région $\mathrm{N}_{2}$ à large bande interdite $\left({ }^{1}\right)$, et que d'autre part le pseudoniveau de Fermi des trous est constant entre $C_{1}$ et $J$, l'intégration de (2) entre le contact ohmique $C_{1}$ et un point d'abscisse $w$ situé entre $\mathrm{J}_{\text {et }} \mathrm{W}_{2}$ permet d'exprimer

( $\left.{ }^{1}\right)$ En effet, pour une même charge électrostatique, la chute de tension dans une zone accumulée est toujours de faible amplitude par rapport à la chute de tension dans une zone dépeuplée. Toutefois, pour que la première soit tout à fait négligeable vis-à-vis de la seconde, il convient que le dopage de la région 1 ne soit pas plus faible que celui de la région 2 . 
$p(w)$ en fonction du potentiel $V(w)$ sous la forme

$$
\ln \frac{p(w)}{N_{\mathrm{v}}(w)}=-\left[\frac{V(w)}{U_{\mathrm{T}}}+\frac{E_{\mathrm{g}}(w)+\chi(w)}{k T}-\frac{E_{\mathrm{g}_{1}}+\chi_{1}}{k T}\right]+\ln \left(\frac{p_{\mathrm{C}_{1}}}{N_{\mathrm{vc}_{1}}}\right)
$$

soit, en utilisant l'expression (4) de la tension de diffusion :

$$
p(w)=\frac{N_{\mathrm{C}_{2}}}{N_{\mathrm{D}_{2}}} \exp \left(\frac{V_{\mathrm{D}}}{U_{\mathrm{T}}}\right) \frac{n_{\mathrm{i}}^{2}(w)}{N_{\mathrm{C}}(w)} \exp \left[-\frac{V(w)}{U_{\mathrm{T}}}+\frac{\chi_{2}-\chi(w)}{k T}\right] .
$$

Le courant $J_{\mathrm{n}}$ s'écrit alors :

$$
J_{\mathrm{n}} \simeq \frac{q D_{\mathrm{n}_{2}} N_{\mathrm{D}_{2}} \exp \left(-\frac{V_{\mathrm{D}}}{U_{\mathrm{T}}}\right)}{\int_{\mathrm{J}}^{\mathrm{W}_{2}} \frac{D_{\mathrm{n}_{2}} N_{\mathrm{C}_{2}}}{D_{\mathrm{n}}(w) N_{\mathrm{C}}(w)} \exp \left[-\frac{V(w)}{U_{\mathrm{T}}}+\frac{\chi_{2}-\chi(w)}{k T}\right] \mathrm{d} w}\left[\exp \left(\frac{V_{\mathrm{a}}}{U_{\mathrm{T}}}\right)-1\right]
$$

Pour donner à l'expression de $J_{\mathrm{n}}$ une forme plus facilement exploitable, il convient d'émettre quelques hypothèses supplémentaires. Nous allons successivement considérer le cas de la jonction abrupte et le cas de la jonction graduelle.

2.3.1 Jonction abrupte. - En considérant que la zone de transition a des limites abruptes et qu'elle est entièrement dépeuplée de porteurs, la loi de variation $V(w)$ du potentiel électrostatique s'écrit :

$$
V(w)=\frac{q N_{\mathrm{D}_{2}}}{2 \varepsilon_{2}}\left(2 w W_{2}-w^{2}\right)
$$

$W_{2}$, qui désigne l'extension de la charge d'espace, dépend de la tension appliquée selon la loi :

$$
W_{2}=\sqrt{\frac{2 \varepsilon_{2}}{q N_{\mathrm{D}_{2}}}\left(V_{\mathrm{D}}-V_{\mathrm{a}}\right)} .
$$

On ne peut calculer la valeur de $J_{\mathrm{n}}$ à partir des relations (28) à (30) que par des méthodes numériques. Nous qualifierons cette procédure de semi-analytique pour la distinguer de la simulation numérique exacte à l'aide du programme complet que nous avons déjà évoqué. Par contre, si l'on approche la variation de potentiel par une loi linéaire, on peut exprimer simplement $J_{\mathrm{n}}$. Pour ce faire, nous pouvons confondre la courbe correspondant à (29) avec sa tangente à l'origine (voir Fig. 6), ce qui revient à considérer que :

$$
V(w)=2\left(V_{\mathrm{D}}-V_{\mathrm{a}}\right) \frac{w}{W_{2}} .
$$

Cette approximation a l'avantage d'être très proche de la réalité au voisinage de la jonction métallurgique, c'est-à-dire là où $p / n_{\mathbf{i}}^{2}$ prend ses plus fortes valeurs et où se situe par conséquent là part la plus influente du "spike». Dans ces conditions et en supposant $\left|V_{\mathrm{D}}-V_{\mathrm{a}}\right|>U_{\mathrm{T}}$, l'équation de la caractéristique

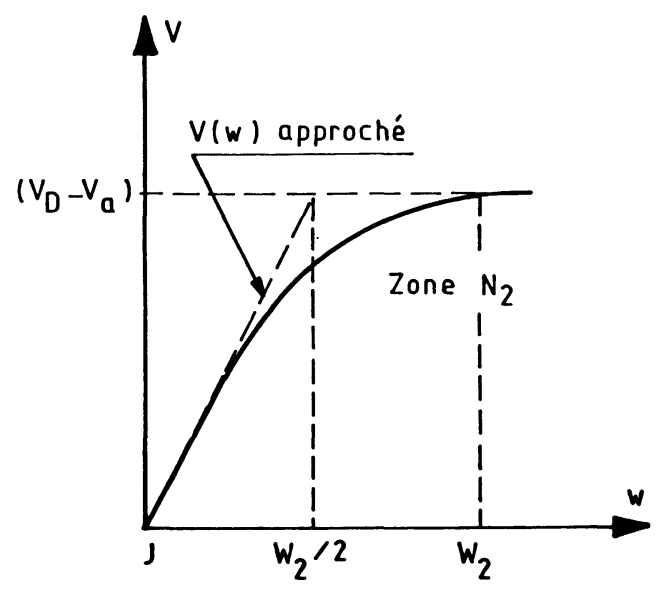

Fig. 6. - Variation du potentiel électrostatique dans la zone dépeuplée.

[Electrostatic potential distribution in a depleted region.]

courant-tension $\left(J_{\mathrm{n}}, V_{\mathrm{a}}\right)$ de l'hétérojonction isotype abrupte s'écrit :

$$
\begin{array}{r}
\left(J_{\mathrm{n}}\right)_{\mathrm{abr} .} \simeq \frac{\mu_{\mathrm{n}_{2}}\left(q N_{\mathrm{D}_{2}}\right)^{3 / 2} \sqrt{2\left(V_{\mathrm{D}}-V_{\mathrm{a}}\right)} \exp \left(-\frac{V_{\mathrm{D}}}{U_{\mathrm{T}}}\right)}{\sqrt{\varepsilon_{2}}} \times \\
\times\left[\exp \left(\frac{V_{\mathrm{a}}}{U_{\mathrm{T}}}\right)-1\right] .
\end{array}
$$

2.3.2 Jonction graduelle. - Nous admettrons dans ce qui suit que la " gradualité » de la largeur de bande interdite du GaAlAs s'étend sur une distance $L_{\mathbf{g}}$ inférieure à l'extension $W_{2}$ de la zone de transition. Nous supposerons par ailleurs que le taux d'aluminium varie de façon linéaire entre 0 et $L_{\mathrm{g}}$ ce qui implique, compte tenu des relations généralement admises dans la littérature entre la largeur de bande interdite et l'affinité électronique d'une part, et la concentration d'aluminium d'autre part, que les quantités $E_{\mathbf{g}}$ et $\chi$ 
sont elles aussi fonctions linéaires de l'abscisse de position $w$. On peut alors écrire :

$$
\left.\begin{array}{c}
E_{\mathbf{g}}(w)=E_{\mathbf{g}_{1}}+\frac{E_{\mathbf{g}_{2}}-E_{\mathbf{g}_{1}}}{L_{\mathbf{g}}} w \\
\chi(w)=\chi_{1}-\frac{\chi_{1}-\chi_{2}}{L_{\mathrm{g}}} w
\end{array}\right\}
$$

Enfin, nous négligerons les conséquences éventuelles que pourraient entraîner les variations des densités effectives d'états $N_{\mathrm{C}}$ et $N_{\mathrm{v}}$, de la permittivité $\varepsilon$ des matériaux et de la constante de diffusion des électrons
$D_{\mathrm{n}}$ dans la zone comprise entre les abscisses 0 et $L_{\mathrm{g}}$. Cette hypothèse est légitime dans la mesure où, d'une part, les valeurs de ces paramètres ne subissent jamais de gros écarts et où, d'autre part, leur impact est toujours de peu de poids par rapport à celui de $\Delta \chi$ ou de $\Delta E_{\mathrm{g}}$ ainsi que cela apparait dans les relations (26) à (28).

Dans ces conditions, et en approchant ici encore la variation de potentiel par la loi linéaire (31), nous pouvons exprimer la caractéristique courant-tension de l'hétérojonction graduelle sous la forme :

$$
\left(J_{\mathrm{n}}\right)_{\mathrm{grad} .}=\eta\left(J_{\mathrm{n}}\right)_{\mathrm{abr}}
$$

avec

$$
\begin{aligned}
\frac{1}{\eta}=\frac{1}{\frac{\chi_{1}-\chi_{2}}{q\left(V_{\mathrm{D}}-V_{\mathrm{a}}\right)} \cdot \frac{W_{2}}{L_{\mathrm{g}}}-1}\left\{\exp \left[-\frac{L_{\mathrm{g}}}{W_{2}} \cdot \frac{\left(V_{\mathrm{D}}-V_{\mathrm{a}}\right)}{U_{\mathrm{T}}}\right]-\exp \left(-\frac{\chi_{1}-\chi_{2}}{U_{\mathrm{T}}}\right)\right\}+ \\
\quad+\exp \left[-\frac{L_{\mathrm{g}}}{W_{2}} \cdot \frac{\left(V_{\mathrm{D}}-V_{\mathrm{a}}\right)}{U_{\mathrm{T}}}\right]-\exp \left(-\frac{V_{\mathrm{D}}-V_{\mathrm{a}}}{U_{\mathrm{T}}}\right) .
\end{aligned}
$$

2.4 Discussion. - Dans le cas de la jonction abrupte, on remarque :

- qu'il n'apparaît pratiquement pas de facteur de non-idéalité $n$ dans la loi de variation de $J_{\mathrm{n}}$ en fonction de la tension appliquée $V_{\mathrm{a}}$, l'influence du terme $\sqrt{V_{\mathrm{D}}-V_{\mathrm{a}}}$ étant tout à fait négligeable par rapport à celle de $\exp \left(V_{\mathrm{a}} / U_{\mathrm{T}}\right)$;

- que, dans l'équation $\left(J_{\mathrm{n}}, V_{\mathrm{a}}\right)$, l'unité n'est pas négligeable par rapport à $\exp \left(V_{\mathrm{a}} / U_{\mathrm{T}}\right)$ sur une large partie du domaine de polarisation, la forte valeur du courant de saturation limitant l'amplitude de la tension directe appliquée à quelques dizaines de $\mathrm{mV}$ seulement.

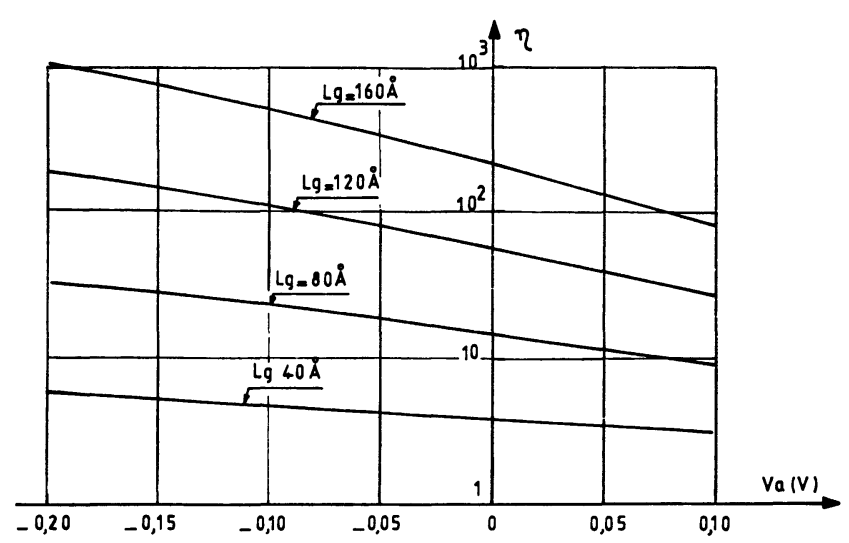

Fig. 7. - Variations du facteur $\eta$ en fonction de la tension appliquée pour différentes valeurs de $L_{\mathrm{g}}$-hétérojonction $\operatorname{GaAs}\left(\mathrm{N}_{1}-10^{19} \mathrm{~cm}^{-3}\right) / \mathrm{Ga}_{0,6} \mathrm{Al}_{0,4} \mathrm{As}\left(\mathrm{N}_{2}-10^{17} \mathrm{~cm}^{-3}\right)$.

[Dependence of factor $\eta$ on applied voltage for different values of $L_{\mathrm{g}}$. Heterojunction $\operatorname{GaAs}\left(\mathrm{N}_{1}-10^{19} \mathrm{~cm}^{-3}\right)$ / $\left.\mathrm{Ga}_{0.6} \mathrm{Al}_{0.4} \mathrm{As}\left(\mathrm{N}_{2}-10^{17} \mathrm{~cm}^{-3}\right) \cdot\right]$
Dans le cas de la jonction graduelle on observe que l'influence du "spike" s'atténue très vite lorsque la gradualité s'étend (fortes valeurs de $\eta$, cf. Fig. 7) ce qui minimise l'effet redresseur qui ne se manifeste alors que pour des densités de courant très élevées.

La comparaison des résultats analytiques que nous venons d'établir avec ceux obtenus à partir de la simulation numérique exacte du comportement d'une hétérojonction isotype est présentée sur les figures 8,9 (structure abrupte) et 10 (structure graduelle).

La concordance obtenue dans le cas des hétérojonctions abruptes est tout à fait convenable; on notera la valeur très élevée du courant inverse qui diminue toutefois lorsque la concentration d'aluminium $x$ augmente et que par conséquent la dissymétrie de la structure est accentuée.

Dans le cas des hétérojonctions graduelles, l'accord entre les résultats analytiques et numériques s'amoindrit lorsque l'extension $L_{\mathbf{g}}$ de la gradualité augmente. Pour interpréter cet écart, il convient de tenir compte de la présence des porteurs libres dont la concentration augmente dans la zone dépeuplée, faussant ainsi les résultats analytiques. Fort heureusement, les valeurs de $L_{\mathbf{g}}$ qui conduisent à un désaccord marqué sont telles que l'influence du "spike " est tout à fait atténuée et le comportement électrique global de la structure est pratiquement ohmique.

A titre d'illustration, nous avons reproduit sur les figures 11,12 et 13 les caractéristiques $(J, V)$ pour différentes structures ainsi que la résistance dynamique $\mathrm{d} V_{\mathrm{a}} / \mathrm{d} J$ qui leur est associée. Ces résultats de simulation correspondent aux comportements expérimentaux de l'hétérojonction isotype $\operatorname{GaAlAs}(\mathrm{N}) / \mathrm{GaAs}(\mathrm{N})$; ils mettent tout particulièrement en évidence l'influence 

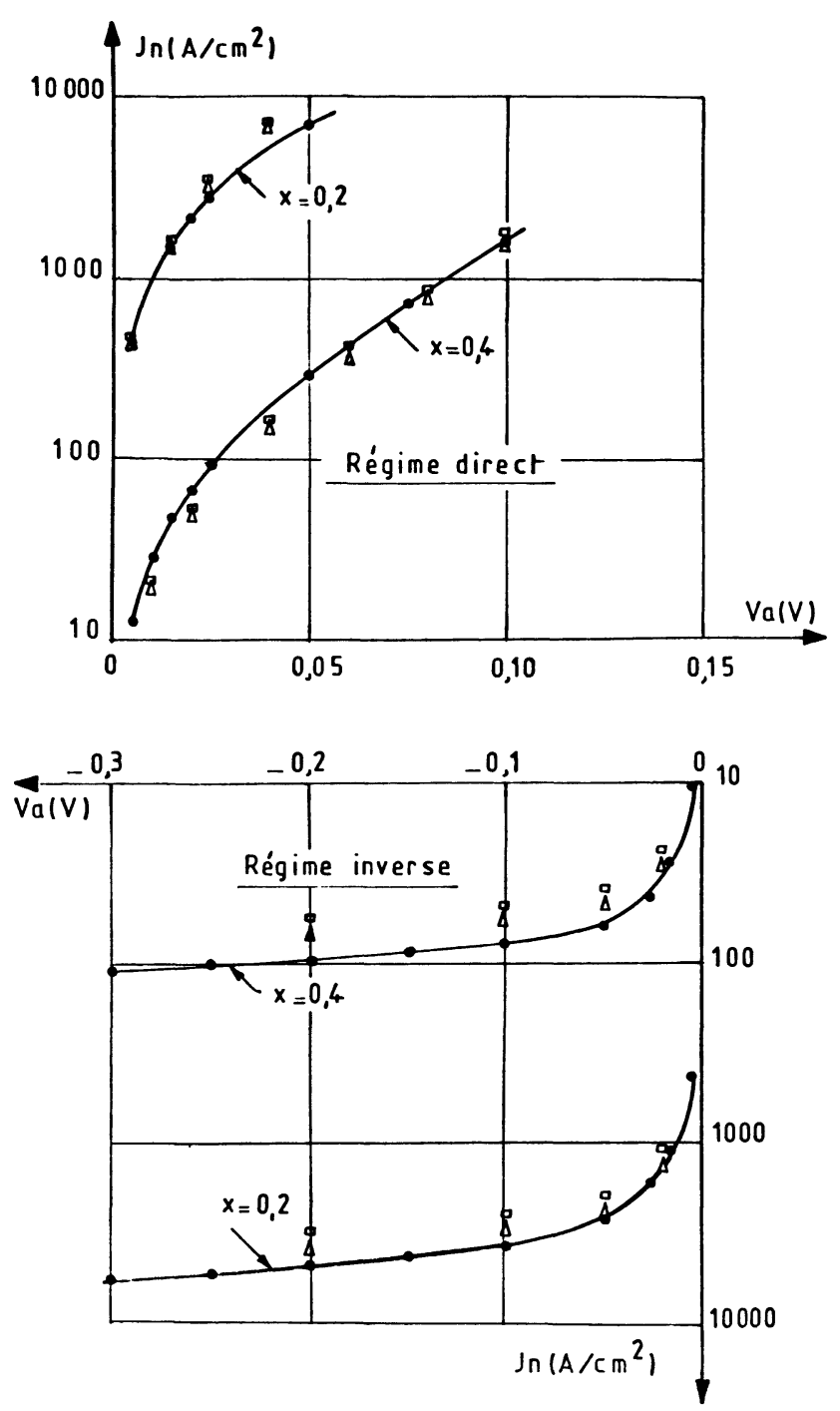

Fig. 9. - Comparaison des caractéristiques $\left(J_{\mathrm{n}}, V_{\mathrm{a}}\right)$ de l'hétérojonction abrupte $\mathrm{GaAs}\left(\mathrm{N}_{1}\right) / \mathrm{Ga}_{0,6} \mathrm{Al}_{0,4} \mathrm{As}\left(\mathrm{N}_{2}\right)$ pour différentes valeurs des dopages $N_{\mathbf{D}_{1}}$ et $N_{\mathbf{D}_{2}}$. (- - Simulation numérique exacte, $\square$ calcul semi-analytique, $\Delta$ calcul analytique).

[Comparison of $\left(J_{n}, V_{\mathrm{a}}\right)$ characteristics of the $\operatorname{GaAs}\left(\mathrm{N}_{1}\right)$ / $\mathrm{Ga}_{0.6} \mathrm{Al}_{0.4} \mathrm{As}\left(\mathrm{N}_{2}\right)$ heterojunction for different values of $N_{\mathbf{D}_{1}}$ and $N_{\mathbf{D}_{2}}$. (- purely numerical simulation, $\square$ semianalytical calcul, $\Delta$ analytical calcul).]
Fig. 8. - Comparaison des caractéristiques $\left(J_{n}, V_{\mathrm{a}}\right)$ de l'hétérojonction abrupte $\operatorname{GaAs}\left(\mathrm{N}_{1}-10^{19} \mathrm{~cm}^{-3}\right) /$ $\mathrm{Ga}_{1-x} \mathrm{Al}_{x} \mathrm{As}\left(\mathrm{N}_{2}-10^{17} \mathrm{~cm}^{-3}\right)$ pour $x=0,2$ et pour $x=0,4$. (- Simulation numérique exacte, $\square$ calcul semi-analytique, $\Delta$ calcul analytique).

[Comparison of $\left(J_{n}, V_{\mathrm{a}}\right)$ characteristics of the abrupt $\operatorname{GaAs}\left(\mathrm{N}_{1}-10^{19} \mathrm{~cm}^{-3}\right) / \mathrm{Ga}_{1-x} \mathrm{Al}_{x} \mathrm{As}\left(\mathrm{N}_{2}-10^{17} \mathrm{~cm}^{-3}\right)$ heterojunction with $x=0.2$ and $x=0.4$. ( - purely numerical simulation, $\square$ semi-analytical calcul, $\Delta$ analytical calcul).]
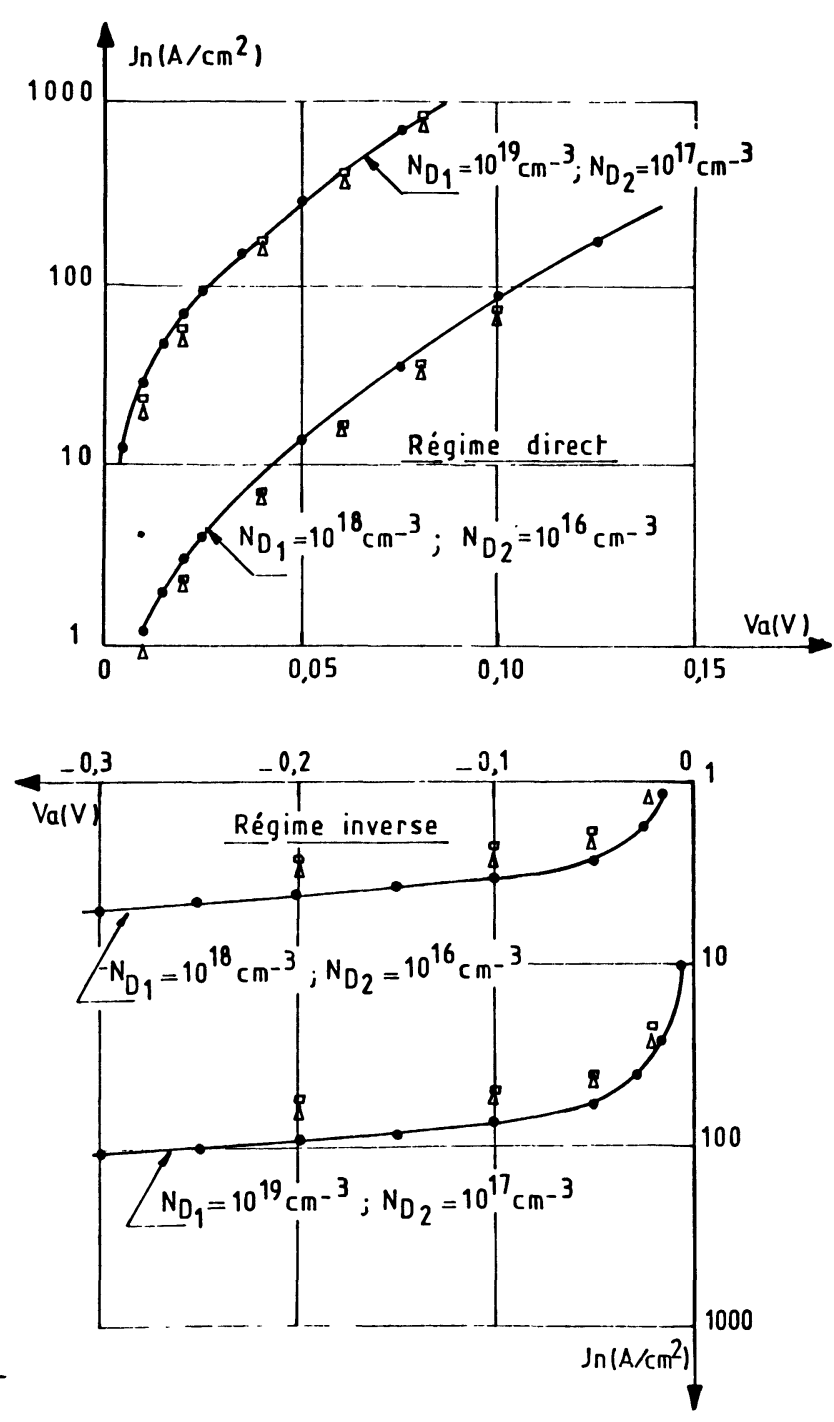

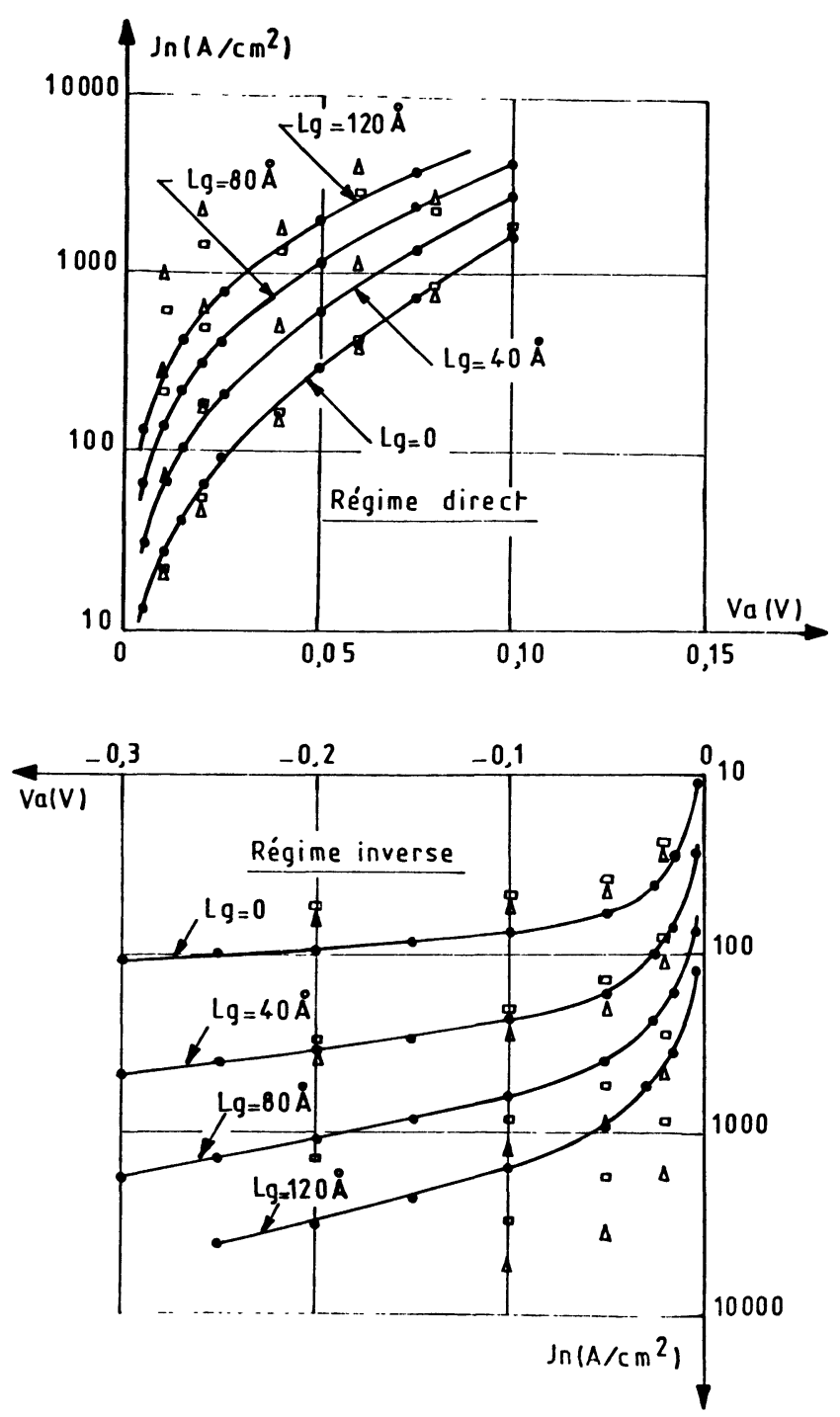

Fig. 11. - Evolutions des caractéristiques $\left(J_{\mathrm{n}}, V_{\mathrm{a}}\right)$ et $\left(\mathrm{d} V_{\mathrm{a}} / \mathrm{d} J_{\mathrm{n}}, J_{\mathrm{n}}\right)$ de l'hétérojonction abrupte $\mathrm{GaAs}\left(\mathrm{N}_{1}\right.$ $\left.10^{19} \mathrm{~cm}^{-3}\right) / \mathrm{Ga}_{1-x} \mathrm{Al}_{x} \mathrm{As}\left(\mathrm{N}_{2}-10^{17} \mathrm{~cm}^{-3}\right)$ pour différentes valeurs de $x(\mathrm{a}-x=0,4 ; \mathrm{b}-x=0,35 ; \mathrm{c}-x=0,3$; $d-x=0,25)$.

[Evolution of the abrupt $\operatorname{GaAs}\left(\mathrm{N}_{1}-10^{19} \mathrm{~cm}^{-3}\right) /$ $\mathrm{Ga}_{1-x} \mathrm{Al}_{x} \mathrm{As}\left(\mathrm{N}_{2}-10^{17} \mathrm{~cm}^{-3}\right)$ heterojunction characteristics $\left(J_{\mathrm{n}}, V_{\mathrm{a}}\right)$ and $\left(\mathrm{d} V_{\mathrm{a}} / \mathrm{d} J_{\mathrm{n}}, J_{\mathrm{n}}\right)$ for different values of $x(\mathrm{a}-x=0.4 ; \mathrm{b}-x=0.35 ; \mathrm{c}-x=0.3 ; \mathrm{d}-x=0.25)$.]
Fig. 10. - Comparaison des caractéristiques $\left(J_{\mathrm{n}}, V_{\mathrm{a}}\right)$ de l'hétérojonction $\mathrm{GaAs}\left(\mathrm{N}_{1}-10^{19} \mathrm{~cm}^{-3}\right) / \mathrm{Ga}_{0,6} \mathrm{Al}_{0,4} \mathrm{As}\left(\mathrm{N}_{2}-\right.$ $\left.10^{17} \mathrm{~cm}^{-3}\right)$ pour différentes valeurs de $L_{\mathrm{g}}$. $\longrightarrow$ - Simulation numérique exacte, $\square$ calcul semi-analytique, $\Delta$ calcul analytique).

[Comparison of $\left(J_{\mathrm{n}}, V_{\mathrm{a}}\right)$ characteristics of the $\mathrm{GaAs}\left(\mathrm{N}_{1}\right.$ $\left.10^{19} \mathrm{~cm}^{-3}\right) / \mathrm{Ga}_{0.6} \mathrm{Al}_{0.4} \mathrm{As}\left(\mathrm{N}_{2}-10^{17} \mathrm{~cm}^{-3}\right)$ for different values of $L_{\mathrm{g}}(--$ purely numerical simulation, $\square$ semianalytical calcul, $\Delta$ analytical calcul).]
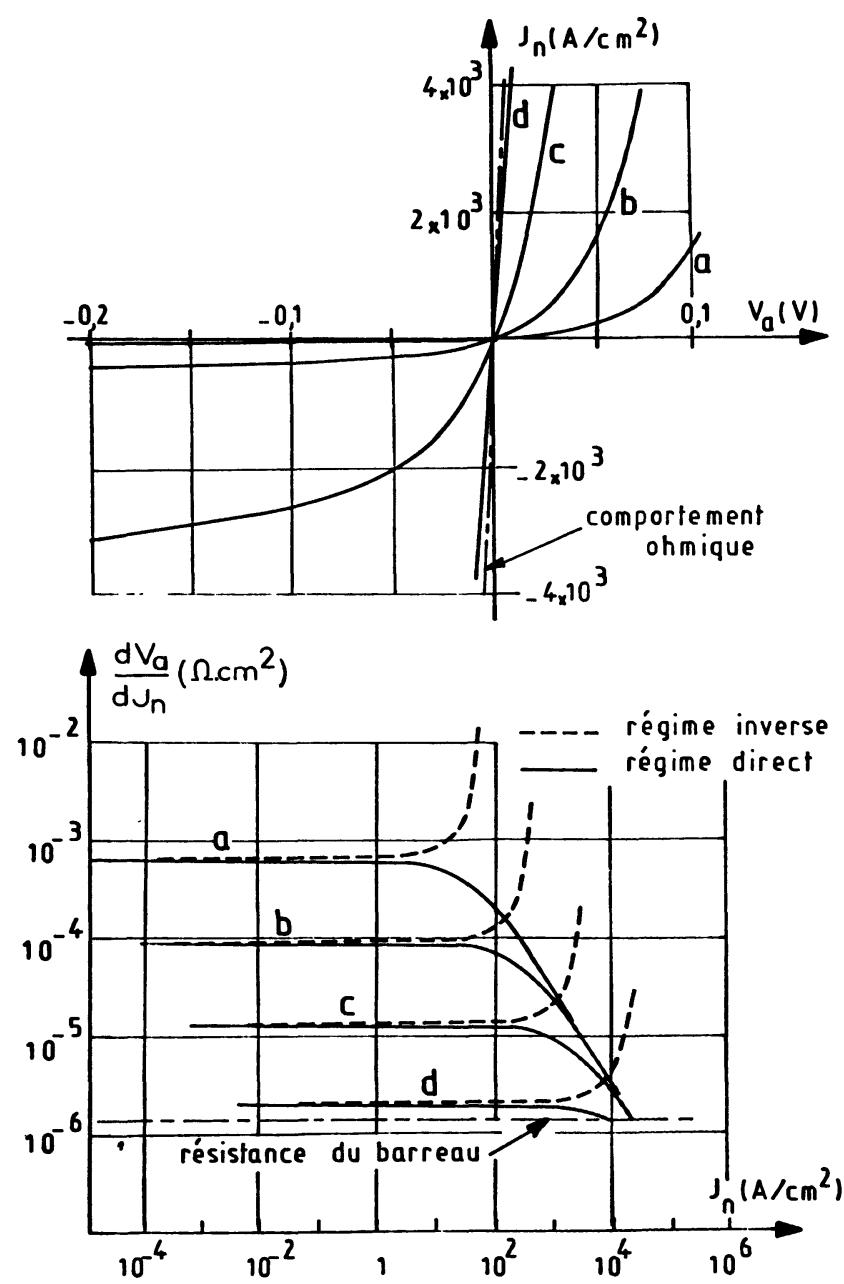

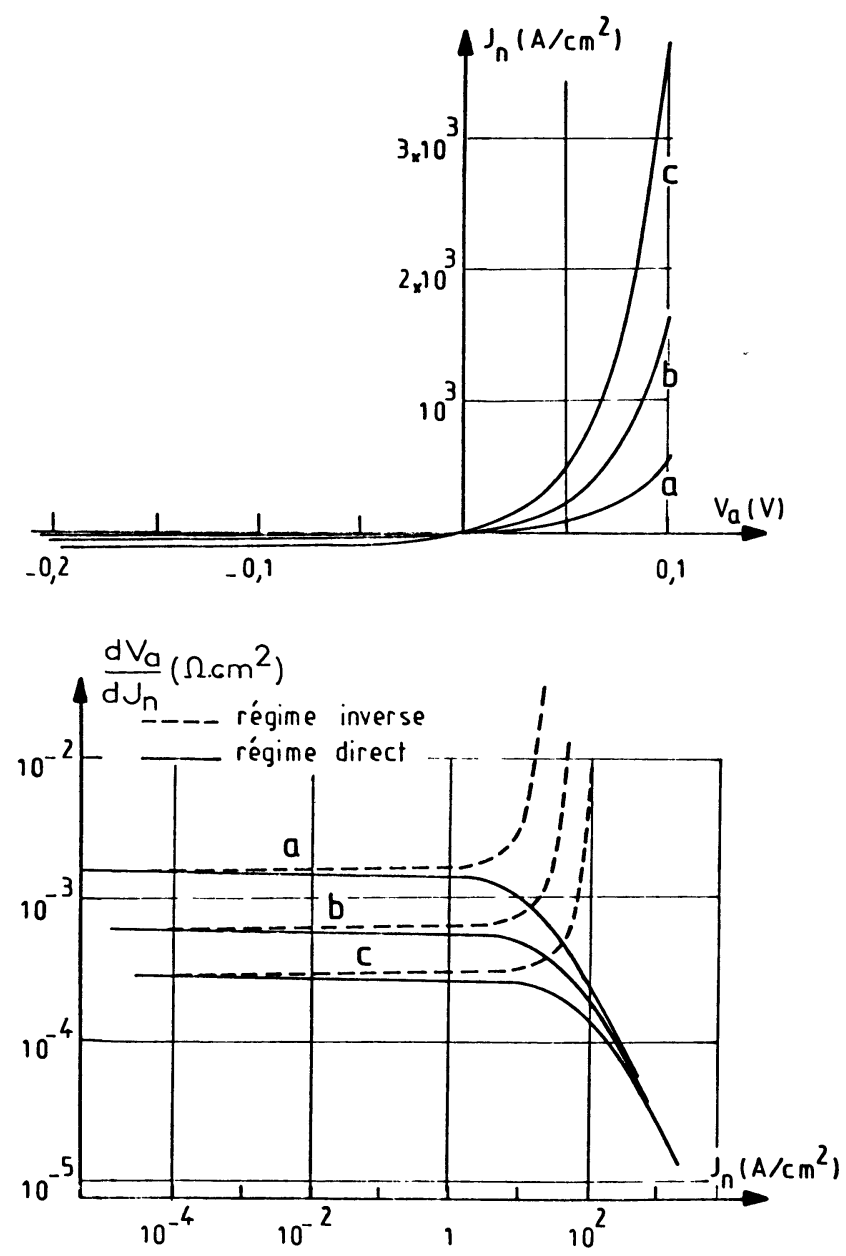

Fig. 13. - Evolutions des caractéristiques $\left(J_{\mathrm{n}}, V_{\mathrm{a}}\right)$ et $\left(\mathrm{d} V_{\mathrm{a}} / \mathrm{d} J_{\mathrm{n}}, J_{\mathrm{n}}\right)$ de l'hétérojonction $\operatorname{GaAs}\left(\mathrm{N}_{1}-10^{19} \mathrm{~cm}^{-3}\right) /$ $\mathrm{Ga}_{0,6} \mathrm{Al}_{0,4} \mathrm{As}\left(\mathrm{N}_{2}-10^{17} \mathrm{~cm}^{-3}\right)$ pour différentes valeurs de $L_{\mathrm{g}} . \quad\left(\mathrm{a}-L_{\mathrm{g}}=0 ; \mathrm{b}-L_{\mathrm{g}}=40 \AA ; \mathrm{c}-L_{\mathrm{g}}=80 \AA\right.$; $\left.\mathrm{d}-L_{\mathrm{g}}=120 \AA: \mathrm{e}-L_{\mathrm{g}}=160 \AA\right)$.

[Evolution of the $\mathrm{GaAs}\left(\mathrm{N}_{1}-10^{19} \mathrm{~cm}^{-3}\right) / \mathrm{Ga}_{0.6} \mathrm{Al}_{0.4} \mathrm{As}\left(\mathrm{N}_{2}-\right.$ $\left.10^{17} \mathrm{~cm}^{-3}\right)$ heterojunction characteristics $\left(J_{\mathrm{n}}, V_{\mathrm{a}}\right)$ and $\left(\mathrm{d} V_{\mathrm{a}} / \mathrm{d} J_{\mathrm{n}}, J_{\mathrm{n}}\right)$ for different values of $L_{\mathrm{g}}\left(\mathrm{a}-L_{\mathrm{g}}=0\right.$; $\mathrm{b}-L_{\mathrm{g}}=40 \AA ; \mathrm{c}-L_{\mathrm{g}}=80 \AA ; \mathrm{d}-L_{\mathrm{g}}=120 \AA ;$ $\left.\mathrm{e}-L_{\mathrm{g}}=160 \AA\right)$.]
Fig. 12. - Evolutions des caractéristiques $\left(J_{n}, V_{\mathrm{a}}\right)$ et $\left(\mathrm{d} V_{\mathrm{a}} / \mathrm{d} J_{\mathrm{n}}, J_{\mathrm{n}}\right)$ de l'hétérojonction abrupte $\operatorname{GaAs}\left(\mathrm{N}_{1}\right.$ $\left.10^{19} \mathrm{~cm}^{-3}\right) / \mathrm{Ga}_{0,6} \mathrm{Al}_{0,4} \mathrm{As}\left(\mathrm{N}_{2}\right)$ pour différentes valeurs de $\mathrm{N}_{\mathrm{D}_{2}} \quad\left(\mathrm{a}-N_{\mathrm{D}_{2}}=10^{16} \mathrm{~cm}^{-3} ; \mathrm{b}-N_{\mathrm{D}_{2}}=10^{17} \mathrm{~cm}^{-3}\right.$; $\mathrm{c}-N_{\mathrm{D}_{2}}=10^{18} \mathrm{~cm}^{-3}$ ).

[Evolution of the abrupt $\operatorname{GaAs}\left(\mathrm{N}_{1}-10^{19} \mathrm{~cm}^{-3}\right)$ / $\mathrm{Ga}_{\mathbf{0 . 6}} \mathrm{Al}_{0.4} \mathrm{As}\left(\mathrm{N}_{2}\right)$ heterojunction characteristics $\left(J_{\mathrm{n}}, V_{\mathrm{a}}\right)$ and $\left(\mathrm{d} V_{\mathrm{a}} / \mathrm{d} J_{\mathrm{n}}, J_{\mathrm{n}}\right)$ for different values of $N_{\mathrm{D}_{2}}\left(\mathrm{a}-N_{\mathrm{D}_{2}}=10^{16} \mathrm{~cm}^{-3}\right.$; $\left.\mathrm{b}-N_{\mathrm{D}_{2}}=10^{17} \mathrm{~cm}^{-3} ; \mathrm{c}-N_{\mathrm{D}_{2}}=10^{18} \mathrm{~cm}^{-3}\right)$.]
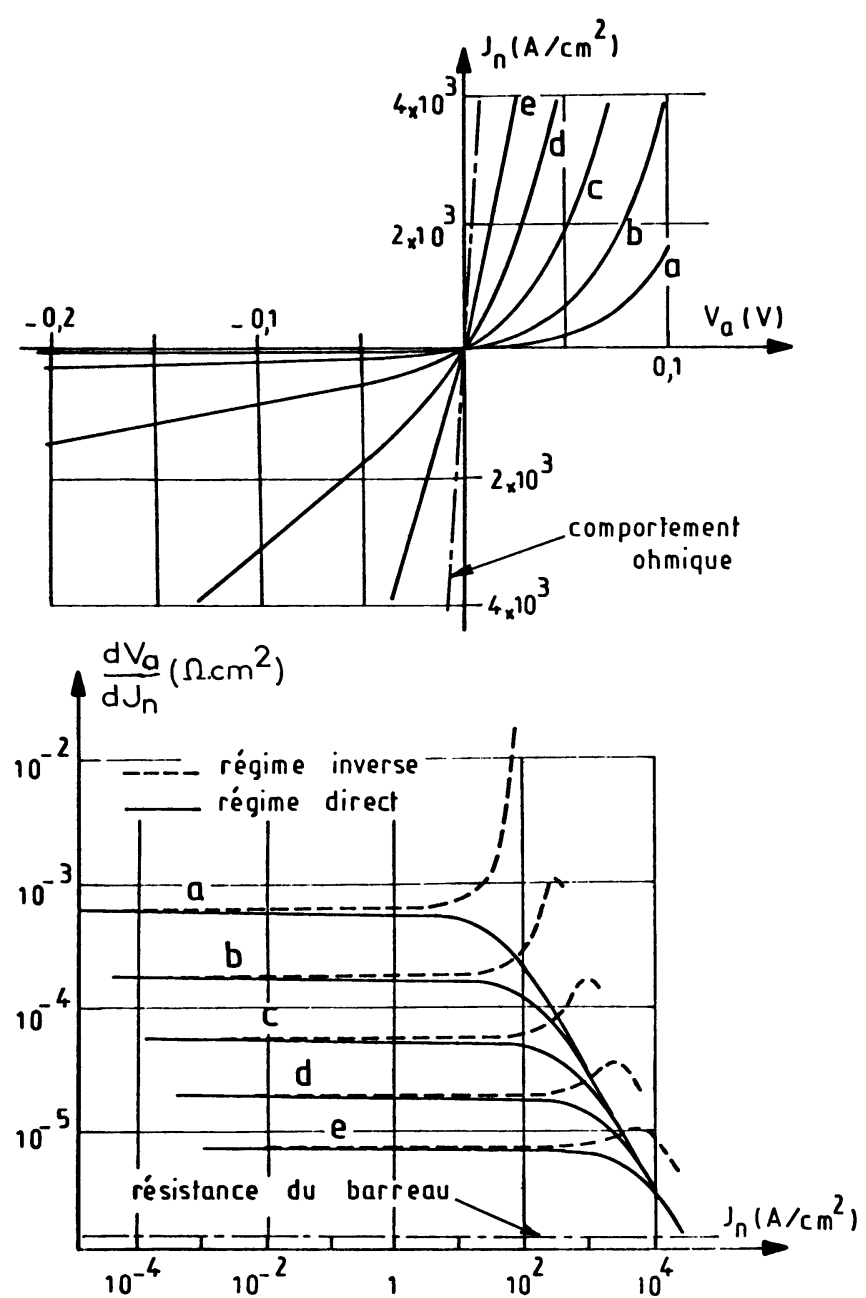


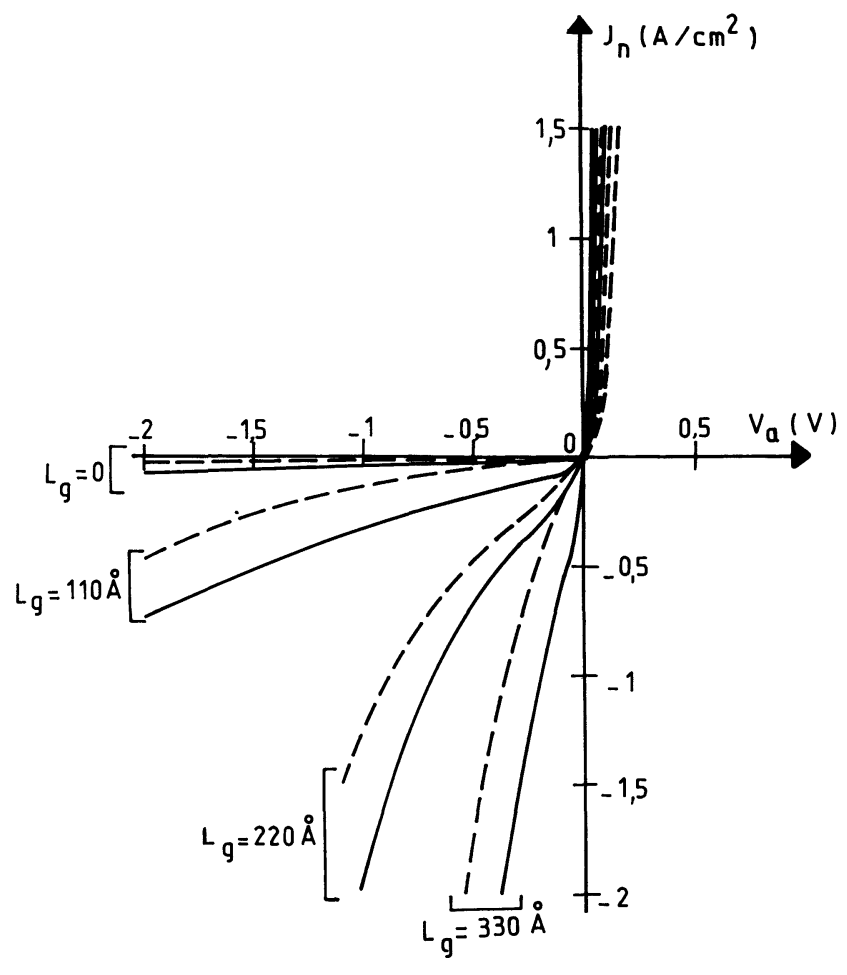

Fig. 14. - Caractéristiques théoriques $\left(J_{n}, V_{\mathrm{a}}\right)$ de l'hétérojonction $\operatorname{GaAs}\left(\mathrm{N}_{1}-10^{17} \mathrm{~cm}^{-3}\right) / \mathrm{Ga}_{0,55} \mathrm{Al}_{0,45} \mathrm{As}\left(\mathrm{N}_{2}-\right.$ $5 \times 10^{15} \mathrm{~cm}^{-3}$ ) pour différentes valeurs de $L_{\mathrm{g}}$. Les traits interrompus ont été calculés par Lee et Pearson [6] à partir de la théorie de l'émission thermoïonique; les lignes continues représentent les résultats déduits de notre modèle $\left(\chi_{1}-\chi_{2}=0,477 \mathrm{eV}, \quad V_{\mathrm{D}}=0,343 \mathrm{~V}, \quad \mu_{\mathrm{n}_{2}}=843 \mathrm{~cm}^{2} / \mathrm{V} . \mathrm{s}\right.$ et $T=297 \mathrm{~K})$.

[Theoretical $\left(J_{\mathrm{n}}, V_{\mathrm{a}}\right)$ characteristics of an $\mathrm{GaAs}\left(\mathrm{N}_{1}-\right.$ $\left.10^{17} \mathrm{~cm}^{-3}\right) / \mathrm{Ga}_{0.55} \mathrm{Al}_{0.45} \mathrm{As}\left(\mathrm{N}_{2}-5 \times 10^{15} \mathrm{~cm}^{-3}\right)$ for different values of $L_{\mathbf{g}}$. The dashed lines are derived from thermoionic emission theory by Lee and Pearson [6]; the solid lines are obtained by our $\operatorname{model}\left(\chi_{1}-\chi_{2}=0.477 \mathrm{eV}\right.$, $V_{\mathrm{D}}=0.343 \mathrm{~V}, \mu_{\mathrm{n}_{2}}=843 \mathrm{~cm}^{2} / \mathrm{V} . \mathrm{s}$ and $\left.T=297 \mathrm{~K}\right)$.]

isotype $\operatorname{GaAlAs}(\mathrm{N}) / \mathrm{GaAs}(\mathrm{N})$. Notre analyse s'est appuyée sur la théorie macroscopique de la diffusion et n'a concerné que des structures idéales supposées sans recombinaisons. Le modèle du type « à contrôle de charge " obtenu, qui prend en compte le caractère plus ou moins graduel du changement de "gap " à la jonction, permet d'exprimer de façon relativement simple, par des relations analytiques compactes, la loi de variation du courant en fonction de la tension appliquée. Nos résultats sont voisins de ceux que prévoient des modèles du type "thermoïonique ».

\section{Bibliographie}

[1] Anderson, R. L., Experiments on Ge-GaAs Heterojunctions. Solid-State Electron. 5 (1962) 341-351.

[2] Oldham, W. G., Milnes, A. G., n-n Semiconductor heterojunctions. Solid-State Electron. 6 (1963) 121-132.

[3] Chandra, A., Eastman, L. F., Rectification at n-n GaAs : (Ga, Al)As heterojunctions. Electron. Lett. 15 (1979) 90-91.

[4] Lechner, A., Kneidinger, M., Planar n-GaAs/NGaAlAs Microwave diodes. Electron. Lett. 16 (1980) 1-2.

[5] De Jaeger, J. C., Salmer, G., Characteristics of isotype n Ge-n GaAs heterojunctions. IEE Proceeding 127 (1980) 207-211.

[6] Lee, S. C., Pearson, G. L., Rectification in $\mathrm{Al}_{x} \mathrm{Ga}_{1-x}$ As-GaAs N-n Heterojunction devices. Solid-State Electron. 24 (1981) 563-568.

[7] Marty, A., Rey, G., Bailbe, J. P., Electrical behavior of an NPN GaAlAs/GaAs heterojunction transistor. Solid-State Electron. 22 (1979) 549-557.
[8] Marty, A., Sur l'étude des transistors bipolaires à hétérojonction $\mathrm{GaAlAs} / \mathrm{GaAs}$ : Théorie et expérience. Thèse de Doctorat d'Etat (Sciences), no 931 , Université Paul Sabatier, Toulouse, juin 1980.

[9] Bailbe, J. P., Marty, A., Pham, H. H., Rey, G. E., Design and fabrication of high-speed $\mathrm{GaAlAs} / \mathrm{GaAs}$ heterojunction transistors. IEEE Trans. Electron Devices ED-27 (1980) 1160-1164.

[10] BeneKING, H., Su, L. M., GaAlAs/GaAs heterojunction microwave bipolar transistor. Electron. Lett. 17 (1981) 301-302.

[11] Lozes-Dupuy, F., Martinot, H., Atallah, K., VasSILIEFF, G., Etude d'une diode au GaAlAs de très forte radiance electroluminescente dans le spectre rouge. Compte rendu de fin d'étude, contrat D.G.R.S.T., mai 1981.

[12] Kressel, H., Lockwood, H. F., Nelson, H., Lowthreshold $\mathrm{Al}_{x} \mathrm{Ga}_{1-x} \mathrm{As}$ visible and IR-light-emitting diode lasers. IEEE J. Quant. Electron. QE-6 (1970) 278-284. 
[13] BaIlbe, J. P., Contribution à l'étude physique des transistors bipolaires. Thèse de Doctorat d'Etat (Sciences), $\mathrm{n}^{\mathrm{0}} 744$, Université Paul Sabatier, Toulouse, 1977

[14] Sutherland, J. E., Hauser, J. R., Computer analysis of heterojunction and graded composition solar cells. IEEE Trans. Electron Devices ED-24 (1977) 363-372.
[15] Leturce, Ph., Rey, G., Physique des composants actîfs à semiconducteurs (Dunod Université) 1978.

[16] Motawie, I., Simulation numérique et modélisation des propriétés des structures électroluminescentes à hétérojonctions multiples. Thèse de Doctorat d'Etat (Sciences) no 891, Université Paul Sabatier, Toulouse, 1979. 